\title{
Evaluation of autofocus functions in molecular cytogenetic analysis
}

\author{
A. SANTOS, ${ }^{*}$ C. ORTIZ DE SOLÓRZANO ${ }^{*} \dagger$ J. J. VAQUERO, ${ }^{*}+$ J. M. PEÑA, ${ }^{*}$ N. MALPICA* \& \\ F. DEL POZO* \\ ${ }^{*}$ Grupo de Bioingeniería y Telemedicina. E.T.S.I. Telecomunicación, Ciudad Universitaria, E-28040 \\ Madrid, Spain \\ †Resource for Molecular Cytogenetics, Lawrence Berkeley National Laboratory, Building 74, 1 \\ Cyclotron Road, Berkeley, CA 94720, U.S.A. \\ $\ddagger$ Nuclear Medicine Department, National Institutes of Health, Bldg 10, Room 1C401, Bethesda, \\ MD 20892-1180, U.S.A.
}

Key words. Analytical cytometry, autofocus, FISH, focusing algorithms.

\begin{abstract}
Summary
This work describes a systematic evaluation of several autofocus functions used for analytical fluorescent image cytometry studies of counterstained nuclei. Focusing is the first step in the automatic fluorescence in situ hybridization analysis of cells. Thirteen functions have been evaluated using qualitative and quantitative procedures. For the last of these procedures a figure-of-merit (FOM) is defined and proposed. This new FOM takes into account five important features of the focusing function. Our results show that functions based on correlation measures have the best performance for this type of image.
\end{abstract}

\section{Introduction}

The automation of fluorescence stained nuclei analysis in cytogenetic studies first requires a reliable, accurate and fast autofocus procedure. Many autofocus algorithms have been proposed and compared in the literature (Groen et al., 1985; Firestone et al., 1991; Yeo et al., 1993; Vollath, 1988; Krotkov, 1987) all of which are based on a criterion function that is applied to images of the same sample acquired with different focuses. The maximum of the function should correspond to the point of optimum focus.

This paper presents a more extensive comparison, and includes some of the most efficient methods published to date. This performance analysis is specific for a particular technique: analytical cytometry using fluorescence in situ hybridization (FISH). As will be seen, the quality of focusing depends on the image content - the larger the information content, the easier the focusing - and the conditions of the

Correspondence to: Andrés Santos; tel: +34 133668 27; fax +34 133668 28; email: andres@die.upm.es image acquisition (noise, existence of objects out of the focus plane, etc.). Owing to the fluorescent nature of FISH images, a specific focusing analysis is needed.

This paper proposes a methodology to assess each focusing function according to a set of criteria that defines its ability to locate the focus position accurately. Apart from accuracy itself and a wide range of applicability, other features are considered to evaluate the performance of a fast focusing procedure. One of these features is the number of images needed, since a large number of acquisitions not only increases the focusing time, but deteriorates the samples faster owing to the bleaching effect. With techniques such as the three-phase method (Boddeke et al., 1994), the focusing point can be located from a reduced set of images, provided the function has two desirable properties: reduced number of false maxima and sharpness of the maximum peak.

The computation time is another feature to be considered. It is important to reduce the total time needed for focusing and thereby to increase the productivity of the system. Although the image analysis and calculations can be performed during the acquisition of the next slide, our experience is that computation can even take longer than the acquisition itself (with our present settings, focusing computations take $1.8 \mathrm{~s}$ per slide, while acquisition is $0.7 \mathrm{~s}$, including $0.5 \mathrm{~s}$ for slide movement). Since focusing has to be repeated a large number of times during the normal processing of a slide, by the end of the analysis it may have a significant effect on the total processing time.

The rest of this paper will first describe both the equipment used and the focusing functions. The next section will discuss the evaluation methodology and the different types of analysis (qualitative, semiquantitative and quantitative). The final two sections will address the results and conclusions. 


\section{Materials and methods}

The focusing algorithms have been tested with real cytogenetic images from DAPI counterstained bone-marrow and peripheral blood cells (provided by Hospital Ramón y Cajal, Madrid) using a Leitz Ergolux microscope (Leitz, Wetzlar, Germany) and a Xillix MicroImager 1400 CCD camera (Xillix, Vancouver, Canada). The objective was a $\times 63$ fluor objective, NA $1 \cdot 30$ (oil immersion). The camera on-chip binning feature (addition of four neighbouring pixels) was used to achieve faster acquisition times with lower light levels, reducing the photobleaching effect.

Under these conditions, $672 \times 519$-pixel images, with 4096 grey levels, are acquired, providing a pixel size of $13.4 \mu \mathrm{m}$, which is approximately double what is required according to the Nyquist sampling theorem. The microscope optical transfer function (OTF) has a cutoff frequency of $5 \cdot 2$ cycles $\mu \mathrm{m}^{-1}$ (green light $\lambda=500 \mathrm{~nm}, \mathrm{NA}=1 \cdot 30$; Boddeke $e t$ al., 1994; Young, 1996). Considering the magnifying factor (63), the Nyquist sampling theorem requires a sampling rate of $6.06 \mu \mathrm{m}$. Although the actual pixel size, $13.4 \mu \mathrm{m}$, is about twice this minimum required sampling rate and thus undoubtedly produces frequency aliasing, its effect can be neglected on the medium spatial frequencies, which is the band in which there is information needed for focusing (see Boddeke et al., 1994; Young, 1996, for a discussion on this topic).

The images were acquired with an exposure time of $0 \cdot 3 \mathrm{~s}$, which produced an average light level of $185 \mathrm{AD}$ units (or ADUs: difference between two consecutive grey levels in the A/D converter).

The achieved depth of focus according to the classical theory (Young, 1996) is $0 \cdot 16 \mu \mathrm{m}$, while the estimated thickness of the objects in this type of FISH study is around $3-5 \mu \mathrm{m}$.

The microscope was fully motorized, $0.025 \mu \mathrm{m}$ being the minimum vertical (focus) step. No special focusing hardware (such as multiple sensors) was used.

The focusing algorithms have been programmed on a SUN/SPARC 4/370 (16 MHz, 12 MB RAM, operating system SunOS $4 \cdot 1.3$ )

\subsection{Autofocus functions}

The autofocus functions give a value that indicates the degree of focusing for each image of the same sample. These functions are based on previous knowledge about the differences in information content in focused and unfocused images. The basic assumption behind most of these functions is that a defocused image results from the convolution of the image with a certain point-spread function (PSF) (Groen et al., 1985), which usually produces a decrease in the high frequencies of the image. This result can also be seen on the assumption that well-focused images contain more information and detail (edges) than unfocused images (Yeo et al. (1993). The focusing functions found in the literature can be classified into five groups: functions based on (1) image differentiation; (2) depth of peaks and valleys; (3) image contrast; (4) histogram and (5) correlation measurements. There is another group of frequency domain functions, but following Yeo et al. (1993), these functions will not be used here as their complexity makes it difficult to produce fast algorithms (Groen et al., 1985; Krotkov, 1987; Firestone et al., 1991; Yeo et al., 1993).

2.1.1. Functions based on image differentiation. Unfocused images usually have slight differences between dark and bright objects, since the PSF distributes each pixel intensity among several pixels, blurring them and averaging their grey levels. Different types of image differentiation have been used as a measure of the focusing of an image.

(1) Thresholded absolute gradient: the first difference is computed and accumulated when it is larger than a certain threshold:

$$
\begin{aligned}
F_{\text {th_grad }}= & \sum_{M} \sum_{N}|g(i, j+1)-g(i, j)| \\
& \text { while }|g(i, j+1)-g(i, j)| \geq v
\end{aligned}
$$

with $g(i, j)$ the grey level intensity of pixel $(i, j)$, and $v$ the gradient threshold.

(2) Squared gradient: similar to the previous function, but squaring the differences, so that the larger gradients have more influence on the final result:

$$
\begin{aligned}
F_{\text {sq_grad }}= & \sum_{M} \sum_{N}|g(i, j+1)-g(i, j)|^{2} \\
& \text { while }|g(i, j+1)-g(i, j)| \geq v .
\end{aligned}
$$

(3) Brenner function: Brenner proposed the use of the first difference between a pixel and its neighbour two points away (Brenner et al., 1971):

$$
\begin{aligned}
F_{\text {brenner }}= & \sum_{M} \sum_{N}|g(i, j+2)-g(i, j)|^{2} \\
& \text { while }|g(i, j+1)-g(i, j)| \geq v .
\end{aligned}
$$

(4) Tenengrad function: Tenenbaum proposed this function described in Yeo et al. (1993) and Krotkov (1987) which uses the Sobel operators to compute the first difference:

$$
\begin{gathered}
F_{\text {tenengrad }}=\sum_{M} \sum_{N} T[g(i, j)] \\
T[g(i, j)]=G_{x}^{2}(i, j)+G_{y}^{2}(i, j)
\end{gathered}
$$

where $T[g(i, j)$ is the square of the gradient value in pixels $(i, j)$ and $G_{x}(i, j)$ and $G_{y}(i, j)$ are the convolution of the image with Sobel operators. Although this function made use of a threshold in its initial form, following Krotkov (1987) no threshold is proposed.

The first three functions $\left(F_{\text {th_grad }}, F_{\text {sq_grad }}, F_{\text {brenner }}\right)$ were slightly modified to consider both the horizontal and the vertical gradients. The maximum was the value used in the 
additions. In a preliminary study, that modification improved the results significantly.

2.1.2. Functions based on depth of peaks and valleys. The depth of peaks and valleys in the grey-level image is another indirect method to estimate the degree of focusing in the image.

(5) Image thresholded content: this function adds the pixel values that are above a threshold (Groen et al., 1985; Mendelsohn \& Mayall, 1972).

$$
\begin{aligned}
F_{\text {th_cont }}= & \sum_{M} \sum_{N} g(i, j) \\
& \text { while } g(i, j) \geq \Theta
\end{aligned}
$$

where $\Theta$ is the intensity threshold.

(6) Thresholded pixel count: this function computes the number of pixels below a threshold (Groen et al. (1985).

$$
\begin{gathered}
F_{\text {num_pix }}=\sum_{M} \sum_{N} s[g(i, j), \Theta] \\
s[x, \Theta]=\left\{\begin{array}{l}
0, x \geq \Theta \\
1, x<\Theta
\end{array}\right.
\end{gathered}
$$

(7) Image power: this computes the sum of the squared values of the pixel intensities:

$$
F_{\text {power }}=\sum_{M} \sum_{N} g(i, j)^{2} \quad \text { while } g(i, j) \geq \Theta .
$$

2.1.3. Functions based on image contrast. The contrast of the image is another feature that characterizes its focusing. It can be estimated with different measures:

(8) Variance: considered by many authors (e.g. Groen et al., 1985; Yeo et al., 1993), it measures the variations in grey level among the image pixels. In this measure, bright and dark pixels have the same influence (with power-based measures, high grey level pixels have more influence than low grey level pixels).

$$
F_{\mathrm{var}}=\frac{1}{M N} \sum_{M} \sum_{N}[g(i, j)-\bar{g}]^{2} .
$$

(9) Normalized variance: this function compensates for the differences in average image brightness among different images:

$$
F_{\text {nor_var }}=\frac{1}{M N \bar{g}} \sum_{M} \sum_{N}[g(i, j)-\bar{g}]^{2} .
$$

2.1.4. Functions based on histogram. These focusing measurements are based on the assumption that focused images have a greater number of grey levels than unfocused images (Firestone et al., 1991).

(10) Range: this is the difference between the maximum and the minimum grey levels. If we denote by $H_{k}$ the number of pixels with intensity $k$, the function computes

$$
F_{\text {range }}=\max \left\{k \mid H_{k}>0\right\}-\min \left\{k \mid H_{k}>0\right\} .
$$

(11) Entropy: entropy (the measure of information content) has also been suggested to evaluate the degree of focusing since, as mentioned before, a focused image has more information content than an unfocused image.

$$
F_{\text {entropy }}=-\sum_{k} p_{k} \log _{2} p_{k}
$$

where $p_{k}$ is the relative frequency of grey level $k$. A threshold is also applied, discarding the smaller intensity values.

2.1.5. Functions based on correlation measures. Vollath (1987, 1988) proposed two focusing measures, with very good performance in the presence of noise, according to the author. One is based on the autocorrelation function and the other on the standard deviation function.

(12) Vollath's $F_{4}$ (Vollath, 1988):

$F_{\text {voll } 4}=\sum_{i=1}^{M-1} \sum_{j=1}^{N} g(i, j) \cdot g(i+1, j)-\sum_{i=1}^{M-2} \sum_{j=1}^{N} g(i, j) \cdot g(i+2, j)$.

(13) Vollath's $F_{5}$ (Vollath, 1988):

$$
F_{\text {voll } 5}=\sum_{i=1}^{M-1} \sum_{j=1}^{N} g(i, j) \cdot g(i+1, j)-M N \bar{g}^{2} .
$$

It should be noted that some of these functions depend on a threshold, while others ( $F_{\text {tenengrad }}, F_{\text {var }}, F_{\text {nor_var }}, F_{\text {range, }}$ $\left.F_{\text {voll4 }}, F_{\text {voll5 }}\right)$ do not depend on any parameter.

Functions $F_{\text {th_grad }}, F_{\text {sq_grad }}, F_{\text {th_cont }}, F_{\text {num_pix }}, F_{\text {power }}, F_{\text {var }}$, $F_{\text {nor_var }}$ were considered in the comparison made by Groen et al. (1985). Functions $F_{\text {brenner }}, F_{\text {var }}, F_{\text {range }}, F_{\text {entropy }}$ were included in the Firestone et al. (1991) analysis. Functions $F_{\text {sq_grad }}, F_{\text {brenner }}, F_{\text {tenengrad }}, F_{\text {var }}$ were analysed by Yeo et al. (1993). Finally, Vollath (1988) studied functions $F_{\text {sq_grad, }}$ $F_{\text {var }}, F_{\text {voll4 }}, F_{\text {voll5 }}$. No study known by the authors made use of all the functions considered in this paper.

\subsection{Evaluation methodology}

Three different methods of evaluation have been followed: qualitative, semiquantitative and quantitative.

2.2.1. Qualitative evaluation. Two series of 81 images were used. In each series, the images from the same object were taken at various distances from the focus point: from $25 \mu \mathrm{m}$ to $+25 \mu \mathrm{m}$ in $0.625-\mu \mathrm{m}$ steps. The two series contain cytogenetic images which are very different in content. The first (Fig. 1, image 4) contains a single nucleus, while the second (Fig. 1, image 2) contains several nuclei, along with isolated chromosomes and debris. These two images were selected specifically since it is well known that performance of the autofocusing systems depends strongly on the image content (a large background 

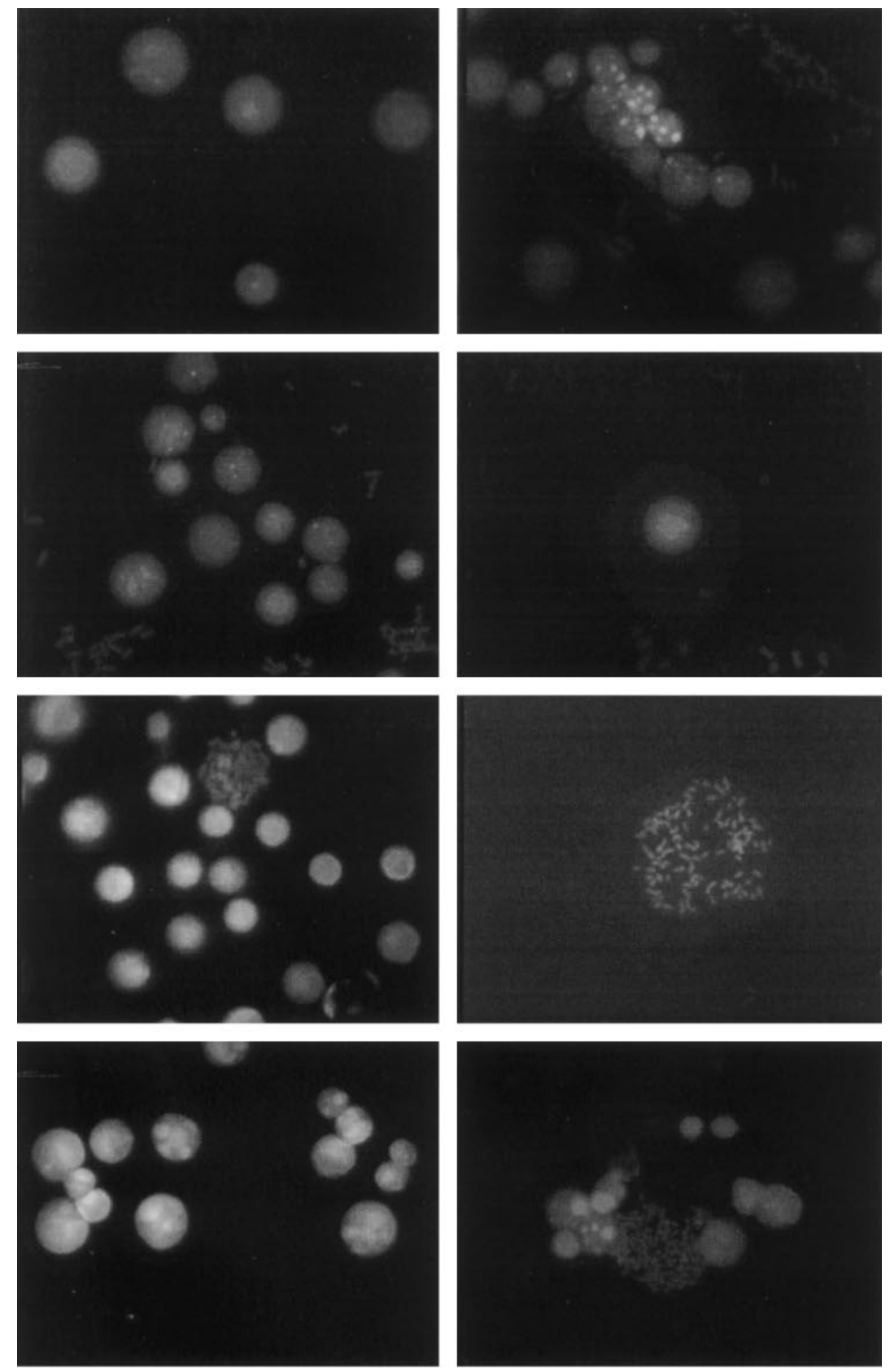

Fig. 1. (Focused) images used in the evaluation. They are referred to as image 1 to image 10 from left to right, top to bottom.
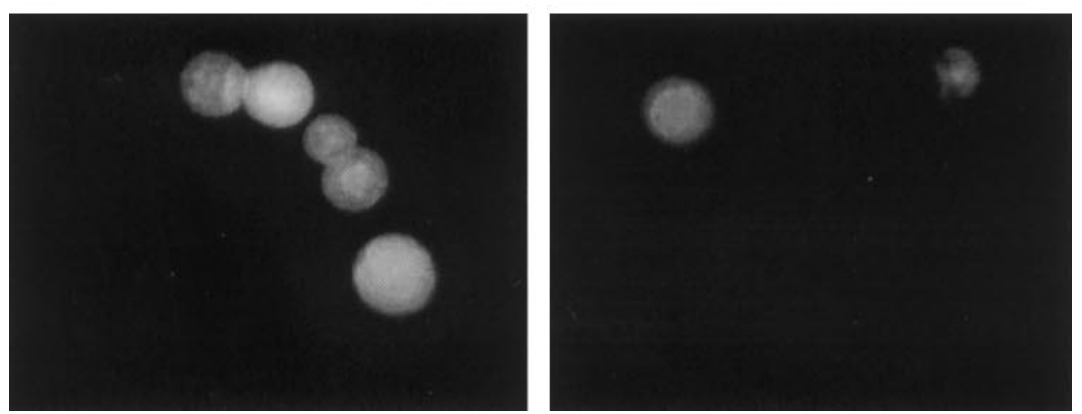
with little image content is more difficult to focus). Between these two series, almost all possible situations are included.

The results for functions $F_{\text {th_cont }}, F_{\text {num_pix }}, F_{\text {power }}, F_{\text {entropy }}$ have been obtained for different intensity thresholds: -90 , $-60, \ldots 300$ plus the average grey level for the focused image. For functions $F_{\text {th_grad }}, F_{\text {sq_grad }}, F_{\text {brenner }}$ the gradient threshold goes from 0 to 65 in steps of 5 .

To reduce the execution time, the focusing function can be computed just for some pixels. Then a sampling period can be defined as the distance between two of these points where the function has been evaluated. A sampling period equal to unity represents that it has been evaluated in every pixel. For each function, the study considers different sampling periods: from 1 to 100 in steps of 5 .

This evaluation allows us to consider, in a visual and intuitive form, the sharpness and position of the focusing maximum, the presence of false maxima and the valid working range.

2.2.2. Semiquantitative (relative) analysis. Following the study by Firestone et al. (1991), this analysis attempts to classify the focusing functions by quantifying several features for each of them. The features considered are:

1 accuracy: difference in steps (in our study each step means $0.625 \mu \mathrm{m}$ ) between the maximum of the focus function and the correct focal position (as obtained by a trained operator);

2 range: interval (measured from the focal position) where the function is monotonically decreasing;

3 number of false maxima;

4 full width at 50\% maximum (FWHM) of the peak in the focus function; the smaller it is, the sharper the focus peak is, and the easier it is to locate it accurately.

5 execution time.

The first four are the features considered in Firestone et al. (1991); the last one (execution time) has been introduced into our analysis.

For this evaluation, 10 series of 81 images each were acquired (Fig. 1). The 13 autofocus functions were applied to each image. The sampling period was equal to unity and all the threshold values considered in the qualitative study were also used.

The functions were ranked with the procedure used in Firestone et al. (1991). For each of the five criteria, a value from 1 to 13 was assigned to each function and image series. The best function was given the value 1 and the worst was 13 . The global function rank score was obtained by adding the scores for each criterion.

To reduce the amount of data presented, only the threshold that produced the best results was included in the tables when considering functions that depended on a threshold. For the same reason, only a sampling period equal to unity was analysed.

This analysis allowed us to order the functions using these five quality criteria, but it did not take advantage of all the available data and did not give an absolute indication of the adequacy of the functions. For this reason, the following quantitative analysis was developed.

2.2.3. Quantitative (absolute) analysis. This more detailed study considered the same five features used in the previous analysis, allowing us to quantify their effect on an individual basis. It consisted of the measure of 'Euclidean distance' that separates each function from the 'ideal' one. This last function was defined as the function that had the following parameters: accuracy, number of false maxima, peak width and execution time all equal to 0 and range equal to 81 (the maximum possible with the 81 image series). To obtain a measure of how each function deviated from this ideal behaviour, the following steps were executed:

1 The mean and the standard deviation of each feature in the 10 image series were obtained.

2 The five feature values for each image series were normalized by subtracting the corresponding mean and dividing by the standard deviation. This produced values for
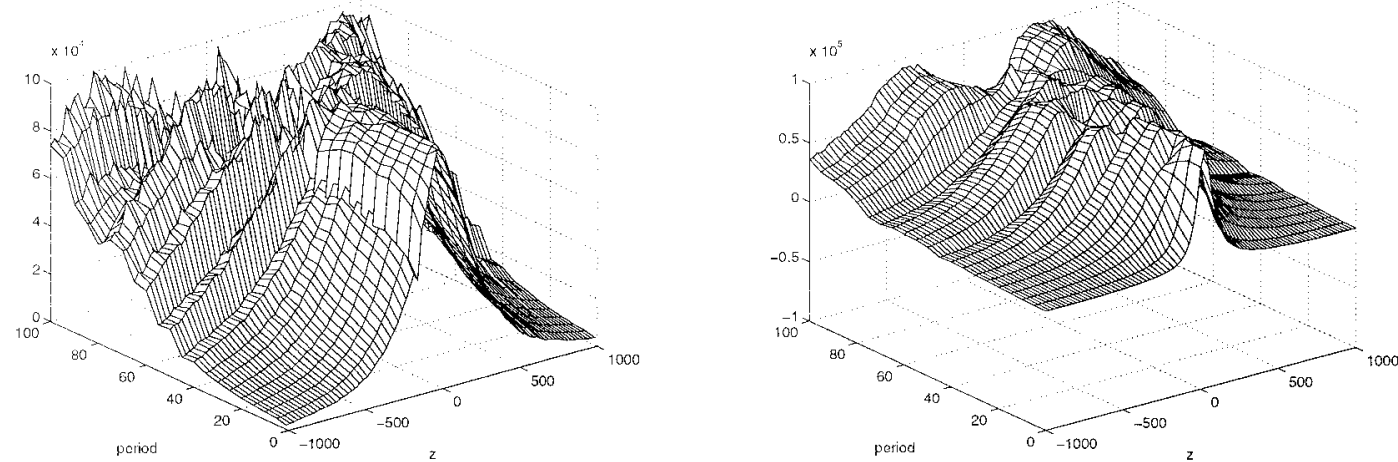

Fig. 2. Qualitative results for function $F_{\text {voll4 }}$, using different sampling periods: left, results for image with low information content (image 4); right, results for image with large information content (image 2). Vertical axis: normalized values of the focus function. Horizontal axis: pixel sampling period and distance to the in-focus position $(z)$. 

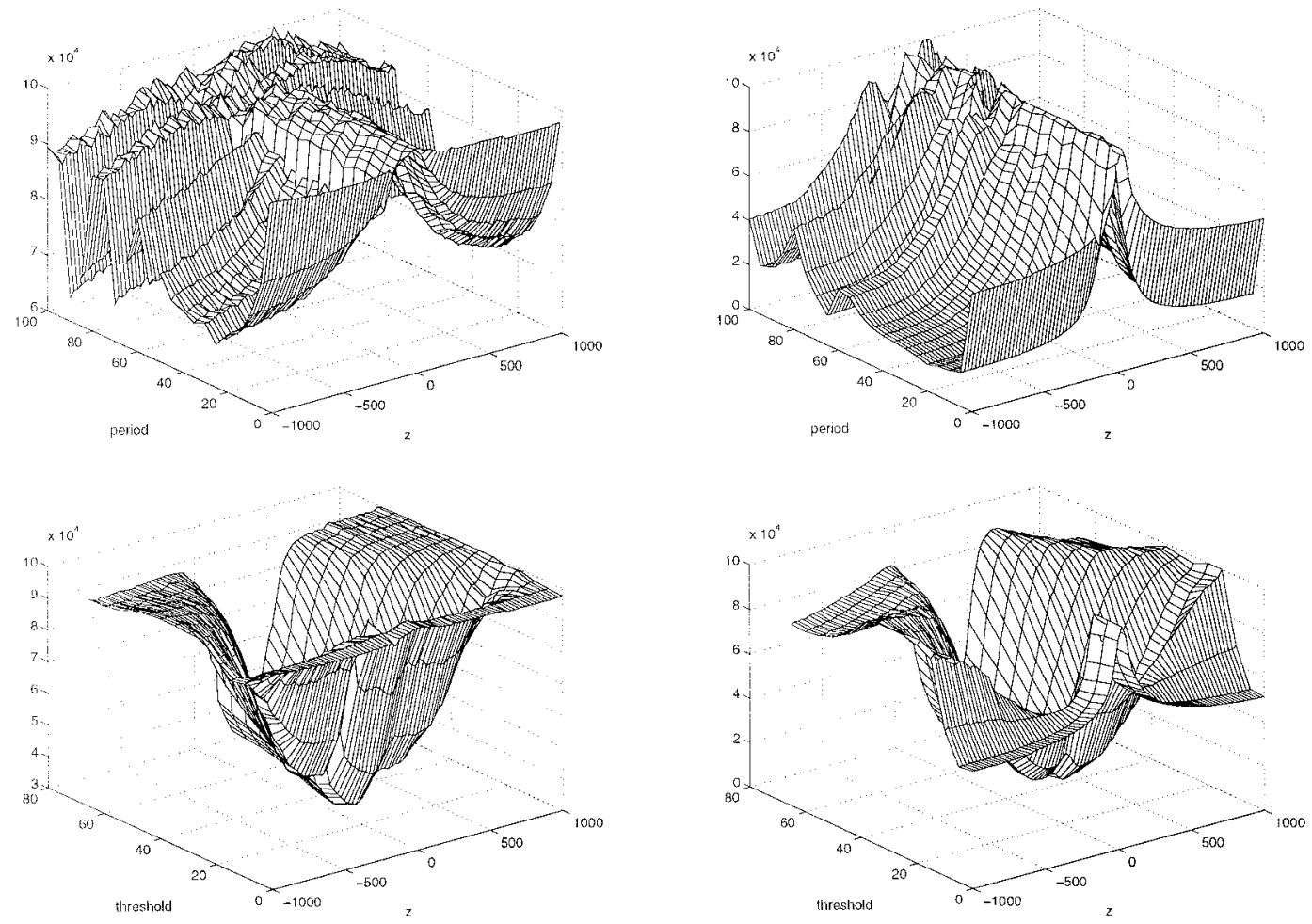

Fig. 3. Qualitative results for function $F_{\text {brenner }}$, using different sampling periods (above) and different thresholds (below): left, results for image with low information content (image 4); right, results for image with large information content (image 2). Vertical axis: normalized values of the focus function. Horizontal axis: pixel sampling period or function threshold, and distance to the in-focus position $(z)$.

the different features that can be compared as they all now have mean zero and standard deviation equal to unity.

3 For each function and for each feature, the distance from the (normalized) ideal function was computed. First, the differences between the feature value for each function and for the ideal function were obtained. Then the square root of the addition of the squares of these results was computed.

4 Finally, to produce a global figure for each function, the mean value of the five distances was obtained.

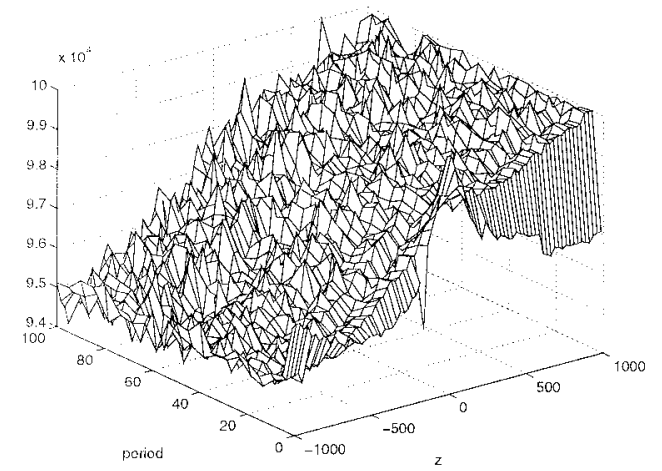

\section{Autofocus evaluation results}

\subsection{Qualitative evaluation}

The results were represented as 3D graphics, where axis $z$ is the normalized value of the focusing function, axis $x$ is the distance from the focusing point (in arbitrary units from -1000 to +1000 , equivalent to $-25 \mu \mathrm{m}$ to $+25 \mu \mathrm{m}$ ) and axis $y$ is either the sampling period or the threshold (gradient threshold for functions $F_{\text {th__rad }}, F_{\text {sq_arad }}, F_{\text {brenner }}$ and intensity

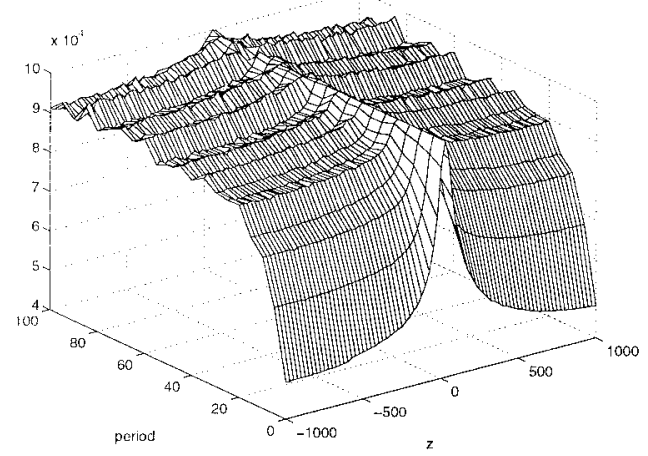

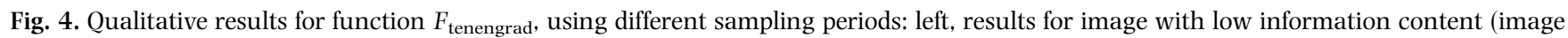
4); right, results for image with large information content (image 2). Vertical axis: normalized values of the focus function. Horizontal axis: pixel sampling period and distance to the in-focus position $(z)$. 

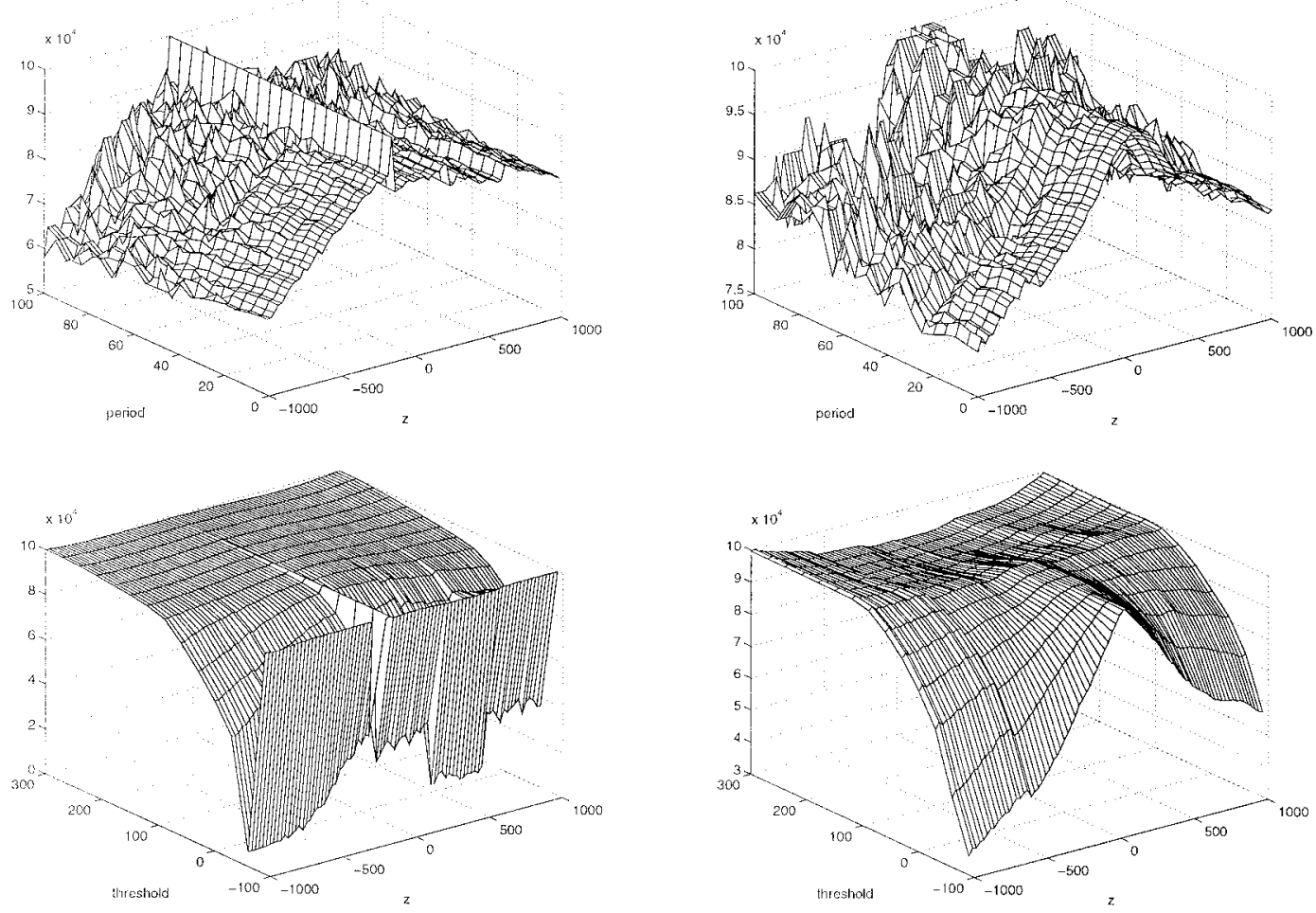

Fig. 5. Qualitative results for function $F_{\text {num_pix }}$, using different sampling periods (above) and different thresholds (below): left, results for image with low information content (image 4); right, results for image with large information content (image 2). Vertical axis: normalized values of the focus function. Horizontal axis: pixel sampling period or function threshold, and distance to the in-focus position $(z)$.

threshold for functions $\left.F_{\text {th_cont }}, F_{\text {num_pix }}, F_{\text {power }}, F_{\text {entropy }}\right)$. Examples of the results for certain focusing functions applied to both test image series are represented in Figs. 2-5.

\subsection{Relative (semiquantitative) evaluation}

Tables 1 and 2 show the results of the focusing functions applied to two illustrative series of images (one with low information content and the other with several nuclei). The tables have been ordered according to the values obtained from the semiquantitative evaluation (starting with the best function). Although the analysis included all the threshold values that were considered in the qualitative evaluation, the tables show only the best result

Table 1. Ordered table of focus functions applied to image 3 (large information content image). Results of semiquantitative evaluation (in parentheses rank for each feature).

\begin{tabular}{|c|c|c|c|c|c|c|c|}
\hline Function & Threshold & Accuracy & Range & False max. & Width & Time (ms) & Score \\
\hline$F_{\text {voll } 4}$ & * & $0(1)$ & $80(1)$ & $1(2)$ & $8(2)$ & 1846 (7) & 13 \\
\hline$F_{\text {voll5 }}$ & * & $2(5)$ & $76(2)$ & $0(1)$ & $18(6)$ & 2677 (10) & 24 \\
\hline$F_{\text {var }}$ & $*$ & $2(5)$ & $76(2)$ & $2(3)$ & $18(6)$ & $2030(9)$ & 25 \\
\hline$F_{\text {num_pix }}$ & -60 & $1(2)$ & $64(7)$ & $4(5)$ & $28(11)$ & $788(2)$ & 27 \\
\hline$F_{\text {nor_var }}$ & $*$ & $2(5)$ & $70(5)$ & $2(3)$ & $26(9)$ & $2028(8)$ & 30 \\
\hline$F_{\text {th_cont }}$ & 240 & $2(5)$ & $28(10)$ & $10(12)$ & $8(2)$ & 704 (1) & 30 \\
\hline$F_{\text {power }}$ & 150 & $2(5)$ & $32(8)$ & $7(9)$ & $12(5)$ & $982(4)$ & 31 \\
\hline$F_{\text {brenner }}$ & 20 & $1(2)$ & $16(12)$ & $4(5)$ & $6(1)$ & $3054(12)$ & 32 \\
\hline$F_{\text {tenengrad }}$ & $*$ & $1(2)$ & $28(10)$ & $7(9)$ & $8(2)$ & 29324 (13) & 36 \\
\hline$F_{\text {sq_grad }}$ & 25 & $3(10)$ & $74(4)$ & $5(7)$ & $24(8)$ & $3020(11)$ & 40 \\
\hline$F_{\text {th_grad }}$ & 5 & $4(11)$ & $70(5)$ & $6(8)$ & $32(12)$ & $1842(6)$ & 42 \\
\hline$F_{\text {entropy }}$ & 210 & $5(12)$ & $32(8)$ & $8(11)$ & $26(9)$ & $1020(5)$ & 45 \\
\hline$F_{\text {range }}$ & * & $*(13)$ & $*(13)$ & 25 (13) & $*(13)$ & 876 (3) & 55 \\
\hline
\end{tabular}


Table 2. Ordered table of focus functions applied to image 9 (small information content image). Results of semiquantitative evaluation (in parentheses rank for each feature).

\begin{tabular}{|c|c|c|c|c|c|c|c|}
\hline Function & Threshold & Accuracy & Range & False max. & Width & Time (ms) & Score \\
\hline$F_{\text {voll4 }}$ & $*$ & $0(1)$ & $80(1)$ & $1(4)$ & $8(2)$ & $1828(7)$ & 15 \\
\hline$F_{\text {num_pix }}$ & 180 & $0(1)$ & $60(8)$ & $0(1)$ & $28(12)$ & $753(2)$ & 24 \\
\hline$F_{\text {nor_var }}$ & $*$ & $-1(6)$ & $78(2)$ & $0(1)$ & $24(8)$ & $1996(8)$ & 25 \\
\hline$F_{\text {tenengrad }}$ & $*$ & $0(1)$ & $62(7)$ & $2(6)$ & $8(2)$ & 29232 & 29 \\
\hline$F_{\text {entropy }}$ & -90 & $2(9)$ & $78(2)$ & $0(1)$ & $38(13)$ & $1033(5)$ & 30 \\
\hline$F_{\text {sq_grad }}$ & 15 & $0(1)$ & $32(11)$ & $2(6)$ & $8(2)$ & 2958 (11) & 31 \\
\hline$F_{\text {brenner }}$ & 10 & $0(1)$ & $58(9)$ & $1(4)$ & $10(6)$ & $3143(12)$ & 32 \\
\hline$F_{\text {th_cont }}$ & 300 & $-1(6)$ & $14(12)$ & $14(12)$ & $6(1)$ & 710 & 32 \\
\hline$F_{\text {power }}$ & 300 & $-2(9)$ & $76(4)$ & $4(10)$ & $24(8)$ & $911(4)$ & 35 \\
\hline$F_{\text {var }}$ & * & $-2(9)$ & $76(4)$ & $3(8)$ & $24(8)$ & $1998(9)$ & 38 \\
\hline$F_{\mathrm{voll} 5}$ & $*$ & $-2(9)$ & $76(4)$ & $3(8)$ & $24(8)$ & $2646(10)$ & 39 \\
\hline$F_{\text {th_grad }}$ & 30 & $-1(6)$ & $10(13)$ & $16(13)$ & $8(2)$ & $1803(6)$ & 40 \\
\hline$F_{\text {range }}$ & * & $10(13)$ & $50(10)$ & $6(11)$ & $29(7)$ & 881 & 44 \\
\hline
\end{tabular}

obtained for each function. For all of them the sampling period is 1 .

Table 3 shows the result of adding the punctuation of each function in the whole set of images.

\subsection{Quantitative absolute evaluation}

Table 4 shows the distance values obtained for each function parameter, along with the average value for each function. In four image series, the $F_{\text {range was so noisy that it }}$ was not possible to determine the function maximum, the peak width or the range. Therefore no value appears for these parameters in the table.

Table 5 shows the same average values that are presented in Table 4, excluding the execution time.

Table 3. Global results of semiquantitative evaluation.

\begin{tabular}{rlr}
\hline & & \\
Ranks & Function & Score \\
\hline 1 & $F_{\text {voll4 }}$ & 147 \\
2 & $F_{\text {nor_var }}$ & 271 \\
3 & $F_{\text {voll5 }}$ & 272 \\
4 & $F_{\text {power }}$ & 281 \\
5 & $F_{\text {var }}$ & 291 \\
6 & $F_{\text {th_cont }}$ & 303 \\
7 & $F_{\text {num_pix }}$ & 320 \\
8 & $F_{\text {th_grad }}$ & 331 \\
9 & $F_{\text {tenengrad }}$ & 336 \\
10 & $F_{\text {entropy }}$ & 363 \\
11 & $F_{\text {brenner }}$ & 375 \\
12 & $F_{\text {sq_grad }}$ & 376 \\
13 & $F_{\text {range }}$ & 434 \\
\hline
\end{tabular}

\section{Discussion}

From the qualitative evaluation, the following conclusions can be deduced.

1 Although, in all cases, the results are much worse with the image with low information content, this worsening is less pronounced in functions $F_{\text {voll4 }}$ and $F_{\text {voll5. }}$. These two functions seem to work properly on cytogenetic images with a small number of nuclei.

2 Considering the existence of false maxima near the absolute maximum, functions based on depth of peaks and valleys $\left(F_{\text {th_cont }}, F_{\text {num_pix }}\right)$ and entropy $\left(F_{\text {entropy }}\right)$ perform badly, while functions $F_{\text {th_grad }}, F_{\text {sq_grad }}, F_{\text {brenner }}, F_{\text {tenengrad }}$, $F_{\text {voll4 }}$ perform well.

3 With respect to the high content images, accuracy is

Table 4. Normalized distance to the ideal function for each function and each parameter (see text), plus average value.

\begin{tabular}{|c|c|c|c|c|c|c|}
\hline Function & Accuracy & Range & False max. & Peak width & Time & Average \\
\hline$F_{\text {voll4 }}$ & $0 \cdot 85$ & $1 \cdot 15$ & $1 \cdot 12$ & $2 \cdot 85$ & $0 \cdot 78$ & $1 \cdot 35$ \\
\hline$F_{\text {voll5 }}$ & $2 \cdot 41$ & $1 \cdot 11$ & $1 \cdot 15$ & $6 \cdot 72$ & $1 \cdot 13$ & $2 \cdot 51$ \\
\hline$F_{\text {nor_var }}$ & $2 \cdot 41$ & 0.92 & $1 \cdot 51$ & $7 \cdot 42$ & $0 \cdot 86$ & $2 \cdot 62$ \\
\hline$F_{\text {var }}$ & $2 \cdot 63$ & $1 \cdot 20$ & $2 \cdot 35$ & $6 \cdot 88$ & $0 \cdot 86$ & $2 \cdot 78$ \\
\hline$F_{\text {power }}$ & $2 \cdot 95$ & $4 \cdot 50$ & $2 \cdot 19$ & $6 \cdot 38$ & $0 \cdot 37$ & $3 \cdot 28$ \\
\hline$F_{\text {brenner }}$ & 2.70 & 6.68 & 1.76 & 5.48 & 1.31 & 3.59 \\
\hline$F_{\text {th_grad }}$ & $4 \cdot 39$ & $5 \cdot 28$ & $3 \cdot 83$ & $4 \cdot 70$ & $0 \cdot 84$ & $3 \cdot 81$ \\
\hline$F_{\text {Sq_grad }}$ & $3 \cdot 25$ & $5 \cdot 45$ & $2 \cdot 65$ & $7 \cdot 04$ & $1 \cdot 26$ & 3.93 \\
\hline$F_{\text {num_pix }}$ & $5 \cdot 12$ & $3 \cdot 95$ & $1 \cdot 74$ & $9 \cdot 42$ & 0.33 & $4 \cdot 11$ \\
\hline$F_{\text {th_cont }}$ & $4 \cdot 00$ & $6 \cdot 67$ & $3 \cdot 20$ & $7 \cdot 70$ & $0 \cdot 30$ & $4 \cdot 37$ \\
\hline$F_{\text {entropy }}$ & $6 \cdot 95$ & $7 \cdot 58$ & $2 \cdot 13$ & $5 \cdot 94$ & 0.43 & $4 \cdot 61$ \\
\hline$F_{\text {tenengrad }}$ & $0 \cdot 85$ & $5 \cdot 38$ & $3 \cdot 71$ & $2 \cdot 74$ & $12 \cdot 49$ & $5 \cdot 03$ \\
\hline$F_{\text {range }}$ & - & - & $11 \cdot 23$ & - & $0 \cdot 37$ & - \\
\hline
\end{tabular}


Table 5. Average distance values excluding the execution time from the analysis.

\begin{tabular}{ll}
\hline & \\
$F_{\text {voll4 }}$ & $1 \cdot 49$ \\
$F_{\text {voll5 }}$ & $2 \cdot 85$ \\
$F_{\text {nor_var }}$ & $3 \cdot 06$ \\
$F_{\text {tenengrad }}$ & $3 \cdot 17$ \\
$F_{\text {var }}$ & $3 \cdot 27$ \\
$F_{\text {power }}$ & $4 \cdot 01$ \\
$F_{\text {brenner }}$ & $4 \cdot 16$ \\
$F_{\text {th_grad }}$ & $4 \cdot 55$ \\
$F_{\text {sq_grad }}$ & $4 \cdot 60$ \\
$F_{\text {num_pix }}$ & $5 \cdot 06$ \\
$F_{\text {th_cont }}$ & $5 \cdot 39$ \\
$F_{\text {entropy }}$ & $5 \cdot 65$ \\
$F_{\text {range }}$ & - \\
\hline
\end{tabular}

highest for function $F_{\text {tenengrad}}$, but this function performs badly when the information content is low.

4 The influence of the sampling rate is very high for function $F_{\text {tenengrad }}$ (it deteriorates quickly when the sampling period increases) and very small on other gradient-based functions

$F_{\text {th_grad }}, F_{\text {sq_grad }}, F_{\text {brenner }}$.

5 The influence of the threshold cannot be established in this qualitative analysis. Quantitative analysis is required to produce definitive results.

From the more accurate quantitative evaluation and to a lesser extent from the semiquantitative evaluation, the strong and weak points of each function can be seen. From this analysis, the functions can be classified in the following groups of decreasing performance:

- $F_{\text {voll } 4}$ : Vollath's $F_{4}$.

- $F_{\text {voll5 }}$ (Vollath's $F_{5}$ ), $F_{\text {nor_var }}$ (normalized variance) and $F_{\text {var }}$ (variance).

- $F_{\text {power }}$ (image power), $F_{\text {brenner }}$ (Brenner), $F_{\text {th_grad }}$ (thresholded absolute gradient), $F_{\text {sq_grad }}$ (squared gradient), $F_{\text {num_pix }}\left(\right.$ thresholded pixel count), $F_{\text {th_cont }}$ (image thresholded content) and $F_{\text {entropy }}$ (entropy)

- $F_{\text {tenengrad }}$ (Tenengrad).

- $F_{\text {range }}$ (range).

It should be noted that when the execution time is not included in the evaluation, the results are identical, with the exception of function $F_{\text {tenengrad, }}$, which has been strongly penalized owing to its execution time that is one order of magnitude higher than the execution time of the remaining functions. If the execution time had been ignored, $F_{\text {tenengrad }}$ would have been in the second group.

It is also worth noting that the best four functions do not depend on any threshold.

\section{Conclusions}

Two important conclusions can be reached from this analysis. First, $F_{\text {voll4 }}$ is the most appropriate function for FISH images. This function was not included in most recent analyses, e.g. Groen et al. (1985), Firestone et al. (1991) and Yeo et al. (1993).

It should also be noted that this function achieves good focusing even with low information content images, that is, images with a small number of nuclei, even with only one. With this capacity in mind, lower concentration specimens can be used for FISH analysis, with the additional advantage that the number of nuclei clusters - always difficult to handle - will be reduced.

Another conclusion from this study is that the proposed quantitative evaluation can give a measure of the adequacy of any function to the set of images analysed. It gives a numerical result that indicates the compromise achieved among the desirable features of the focusing function.

\section{Acknowledgments}

We wish to thank Dr José Miguel García-Sagredo and Isabel Vallcorba from Hospital Ramón y Cajal (Spain) for providing the samples used in this study. The research also benefited from the European Union Concerted Action on 'Automation of Molecular Cytogenetic Analysis' (ERBCIPD-CT94-0252) and the Regional Government Health Research Action 07/ 059/96. J.J.V. acknowledges the support of CICYT (Spanish Government). Finally we would like to thank Patrick L. Hoopes for his support in the revision of the final version of this paper.

\section{References}

Boddeke, F.R., van Vliet, L.J., Netten, H. \& Young, I.T. (1994) Autofocusing in microscopy based on the OTF and sampling. Bioimaging, 2, 193-203.

Brenner, J.F., Dew, B.S., Horton, J.B., King, J.B., Neirath, P.W. \& Sellers, W.D. (1971) An automated microscope for cytologic research. J. Histochem. Cytochem. 24, 100-111.

Firestone, L., Cook, K., Culp, K., Talsania, N. \& Preston, K. (1991) Comparison of autofocus methods for automated microscopy. Cytometry, 12, 195-206.

Groen, F., Young, I.T. \& Ligthart, G. (1985) A comparison of different focus functions for use in autofocus algorithms. Cytometry, 12, 81-91.

Krotkov, E. (1987) Focusing. Int. J. Computer Vision, 1, 223-237.

Mendelsohn, M.L. \& Mayall, B.H. (1972) Computer-oriented analysis of human chromosomes - III focus. Comput. Biol. Med. 2, 137.

Vollath, D. (1987) Automatic focusing by correlative methods. J. Microsc. 147, 279-288.

Vollath, D. (1988) The influence of the scene parameters and of noise on the behaviour of automatic focusing algorithms. $J$. Microsc. 151, 133-146.

Yeo, T., Ong, S. \& Jayasooriah, Sinniah, R. (1993) Autofocusing for tissue microscopy. Image Vision Comput. 11, 629-639.

Young, I.T. (1996) Quantitative microscopy. IEEE Eng. Med. Biol. Mag. 15, 59-66. 